This is an electronic reprint of the original article. This reprint may differ from the original in pagination and typographic detail.

Author(s): Koskinen, Pekka; Fampiou, loanna; Ramasubramaniam, Ashwin

Title: Density-Functional Tight-Binding Simulations of Curvature-Controlled Layer Decoupling and Band-Gap Tuning in Bilayer MoS2

Year: $\quad 2014$

Version:

Please cite the original version:

Koskinen, P., Fampiou, I., \& Ramasubramaniam, A. (2014). Density-Functional TightBinding Simulations of Curvature-Controlled Layer Decoupling and Band-Gap Tuning in Bilayer MoS2. Physical review letters, 112(18), Article 186802. https://doi.org/10.1103/PhysRevLett.112.186802

All material supplied via JYX is protected by copyright and other intellectual property rights, and duplication or sale of all or part of any of the repository collections is not permitted, except that material may be duplicated by you for your research use or educational purposes in electronic or print form. You must obtain permission for any other use. Electronic or print copies may not be offered, whether for sale or otherwise to anyone who is not an authorised user. 


\title{
Density-Functional Tight-Binding Simulations of Curvature-Controlled Layer Decoupling and Band-Gap Tuning in Bilayer $\mathrm{MoS}_{2}$
}

\author{
Pekka Koskinen, ${ }^{1, *}$ Ioanna Fampiou, ${ }^{2}$ and Ashwin Ramasubramaniam ${ }^{2, \dagger}$ \\ ${ }^{1}$ NanoScience Center, Department of Physics, University of Jyväskylä, 40014 Jyväskylä, Finland \\ ${ }^{2}$ Department of Mechanical and Industrial Engineering, University of Massachusetts Amherst, \\ Amherst, Massachusetts 01003, USA
}

(Received 15 November 2013; published 8 May 2014)

\begin{abstract}
Monolayer transition-metal dichalcogenides (TMDCs) display valley-selective circular dichroism due to the presence of time-reversal symmetry and the absence of inversion symmetry, making them promising candidates for valleytronics. In contrast, in bilayer TMDCs both symmetries are present and these desirable valley-selective properties are lost. Here, by using density-functional tight-binding electronic structure simulations and revised periodic boundary conditions, we show that bending of bilayer $\mathrm{MoS}_{2}$ sheets breaks band degeneracies and localizes states on separate layers due to bendinginduced strain gradients across the sheets. We propose a strategy for employing bending deformations in bilayer TMDCs as a simple yet effective means of dynamically and reversibly tuning their band gaps while simultaneously tuning valley-selective physics.
\end{abstract}

DOI: 10.1103/PhysRevLett.112.186802

PACS numbers: 73.22.-f

Transition-metal dichalcogenides (TMDCs)—notably, the Group-VI dichalcogenides-have been the subject of much recent interest for nanoscale electronics and photonics [1-5]. Single- and few-layer $M X_{2}(M=\mathrm{Mo}, \mathrm{W} ; X=\mathrm{S}$, $\mathrm{Se}, \mathrm{Te}$ ) compounds, unlike graphene, display appreciable electronic band gaps [4-7], making them promising candidates for nanoscale digital electronics [8,9]. Upon thinning multilayer sheets to monolayer thickness, TMDCs undergo an indirect-to-direct gap transition leading to strong and stable photoluminescence, which is attractive for optoelectronic applications [4,6,7,10]. In addition, recent experiments and simulations have shown that the electronic and optical properties of few-layer TMDCs can be controllably modified by external fields $[11,12]$ and mechanical strains [13-18]. The combination of chemical composition, dimensionality, and external perturbations thus provides a rich space of parameters for designing optimized TMDC nanostructures for device applications.

TMDCs also allow for more complex manipulation of their electronic properties. The band edges of monolayer Group-VI TMDCs lie at the corners of their hexagonal Brillouin zones, conventionally called $K$ and $K^{\prime}$ points, or valleys. Since the monolayer lacks inversion symmetry, the $K$ and $K^{\prime}$ valleys are related only by time-reversal symmetry, which, in conjunction with spin-orbit splitting of the band edges, associates a distinct magnetic moment with each valley [19]. This property enables selective excitation of each valley by circularly polarized light, as demonstrated on $\mathrm{MoS}_{2}$ [20-22] and $\mathrm{WSe}_{2}$ [23] monolayers. In contrast, bilayer TMDCs lose this valley-selective circular dichroism because of the presence of both inversion and time-reversal symmetries. As these symmetries are retained under homogeneous mechanical strains, such strains cannot be used to break the valley degeneracy. This is unfortunate since strain has been shown to alter the band gap by as much as $\sim 120 \mathrm{meV} / \%$ strain in bilayer $\mathrm{MoS}_{2}$ [14]. Finding a way to break valley degeneracy in strained bilayers would be desirable, as it would enable reversible and simultaneous control over the optical band gap and valley-selective phenomena.

Fortunately, there are options for breaking inversion symmetry in bilayer TMDCs. The first trick is to apply a perpendicular electric field; this enables external control over the valley magnetic moment [24,25], while giving the charge carriers an additional degree of freedom, a pseudospin, corresponding to separate layers in the sheet [25-27]. While calculations suggest that the external field can also tune the band gap [11], experimental realization might be difficult as the required fields are rather large. Mechanical strains, however, need only be modest to induce appreciable band-gap tuning. Hence, in this Letter we introduce a second trick, breaking the symmetry by inhomogeneous strains due to uniform bending. From elementary Euler-Bernoulli kinematics, bending imposes a linear variation in strain normal to the layers [28]. Should interlayer coupling be absent, each layer would then be an independent monolayer whose band gap would correlate directly with the strain in that layer. In this ideal picture, the $K$ and $K^{\prime}$ valleys of each layer could be independently excited with photons of specific energy and helicity.

Could this idealized scenario be realized in layered materials such as $\mathrm{MoS}_{2}$ with weak but certainly nonzero interlayer coupling? We address this question in detail via density-functional tight-binding simulations that employ revised periodic boundary conditions to model the presence of curvature in bilayer $\mathrm{MoS}_{2}$. We show that curvature can 
induce complete decoupling of layers around $K\left(L^{\prime}\right)$ and $K^{\prime}(L)$ points, meaning that bands localize on separate layers and their energy shifts are governed by layer-specific strains. Each bent layer then effectively behaves as an independent monolayer, suggesting that distinct valleys in distinct layers could indeed be excited independently. These results suggest that curvature could provide a simple yet effective strategy for simultaneous control over band gaps and valley polarizations in few-layer TMDCs.

To model the electronic structure, we used the densityfunctional tight-binding (DFTB) method with an established set of Mo and S parameters [29,30]. The electronic structures of TMDCs are known to be computationally challenging due to their prominent spin-orbit and quasiparticle features [10,31], and thus they are difficult to model even with density-functional theory (DFT). However, it has been shown that the movement of band edges upon strain follows the same electromechanical trends, regardless of the level of theory, be it DFT or beyond [32]. Most important, these very same electromechanical trends are also reproduced by DFTB [30,33], and validated against DFT even for transport properties [34]. Therefore, DFTB suffices well for the purpose of our work, which is not dependent on high accuracy of numbers but on correct electromechanical trends. For completeness, however, we further validated the method by comparing it explicitly to DFT results for the strain-dependence of the true gap (indirect) and direct gap (at $K$ and $K^{\prime}$ ) for bilayer $\mathrm{MoS}_{2}$ under equibiaxial strain (see Supplemental Material [35]).

Efficient simulation of the bending deformations themselves was enabled by revised periodic boundary conditions [36-39]. Conventionally, a flat sheet would be simulated by the minimal unit cell spanned by vectors $\mathbf{a}_{1}$ and $\mathbf{a}_{2}$ (in the $x y$ plane), where $\left|\hat{a}_{1} \times \hat{a}_{2}\right|=\sin (\pi / 3)$ and lattice constant $a=\left|\mathbf{a}_{i}\right|=3.16 \AA$ A. Here, instead of creating periodic images by the usual translations along $\mathbf{a}_{1}$ and $\mathbf{a}_{2}$, rotations were also used. First, cylindrical deformation was modeled by taking one translation (by $\mathbf{a}_{1}$ ) and one rototranslation (translation by $\mathbf{a}_{1} \cos (\pi / 3)$ followed by rotation of an angle $\alpha=a \sin (\pi / 3) / R$ around $\hat{a}_{1}$, where $R$ is the radius of curvature). Second, spherical deformation was modeled by taking two distinct rotations with respect to axes $\hat{b}_{1}$ and $\hat{b}_{2}$ by an angle $\alpha=a / R$, where $\hat{b}_{i} \times \hat{z}=\hat{a}_{i}$ [40]. Apart from the symmetry operations, the simulations proceeded as for conventional periodic slabs. We note that in the limit of small curvature, $a$ is small compared to $R$ and the unit cell properties can thus be viewed as local properties. We therefore emphasize that a notion about an overall geometrical structure such as "cylinder" or "sphere" is irrelevant [41]. The deformations are illustrated in Fig. 1.

The curvature is quantified by the dimensionless parameter $\Theta=H /(2 R)$, where $H=6.2 \AA$ is the distance between Mo-Mo planes in the bilayer. This parameter is
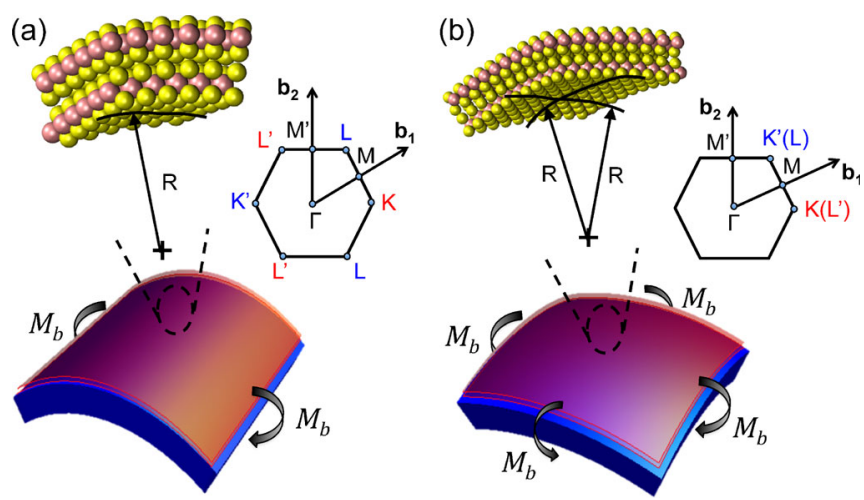

FIG. 1 (color online). Schematic of bilayer $\mathrm{MoS}_{2}$ (purple sheets) on a flexible substrate with (a) cylindrical and (b) spherical curvature, as induced by applied moments $M_{b}$. Insets show the corresponding Brillouin zones and high-symmetry points in reciprocal space. $K$ and $L^{\prime}$, and $K^{\prime}$ and $L$ valleys are equivalent, differing by a reciprocal lattice vector.

convenient because it equals the average tensile strain in the outer layer $\left(\varepsilon_{\text {out }}=\Theta\right)$ and the average compressive strain in the inner layer $\left(\varepsilon_{\mathrm{in}}=-\Theta\right)$, independent of sheet thickness [42]. Finally, for each enforced $\Theta$, the structures were optimized using $10 \times 10 \kappa$ points and a maximum force criterion of $0.01 \mathrm{eV} / \AA$ [40].

Let us first consider the effect of bending on the electronic structure of bilayer $\mathrm{MoS}_{2}$. The first central result of this work is that bending not only alters the band gap but also breaks the band degeneracy as a result of broken layer symmetry [Fig. 2]. This is in sharp contrast to the homogeneous in-plane strains which, as noted earlier, can only change the band gap but cannot break the layer degeneracy [Fig. 2 (top row)]. The bending-induced changes can be understood by considering the orbital composition of the bands. States at the valence band (VB) maximum at $\Gamma$ are dominated by Mo $d_{z^{2}}$ and $\mathrm{S} p_{z}$ orbitals while those at the conduction band $(\mathrm{CB})$ minimum at $K / K^{\prime}$ are mostly comprised of Mo $d_{z^{2}}$ with some mixing of $\mathrm{S} p_{x}$ and $p_{y}[11,43,44]$. Stacking of $\mathrm{MoS}_{2}$ layers confines the diffuse, out-of-plane Mo $d_{z^{2}}$ and $\mathrm{S} p_{z}$ states and increases their energy, causing the direct-to-indirect gap transition from monolayer to multilayer $\mathrm{MoS}_{2}$ [45]. It is precisely these $\operatorname{Mo} d_{z^{2}}$-dominated regions near the $\Gamma$ point of the VB and $K\left(L^{\prime}\right) / K^{\prime}(L)$ points of the $\mathrm{CB}$ that yield the greatest sensitivity to strain-induced lifting of degeneracy upon bending (Fig. 2). On the other hand, due to primary composition of in-plane Mo $d_{x^{2}-y^{2}}$ and Mo $d_{x y}$ orbitals together with a small mixture of $\mathrm{S} p_{x}$ and $p_{y}$ orbitals $[11,43,44]$, the VB maxima at $K\left(L^{\prime}\right) / K^{\prime}(L)$ are more resilient against bending. Moreover, the effect of bending for given $\Theta$ is larger for spherical bending than for cylindrical bending, in agreement with analogous effects seen in equibiaxial versus uniaxial in-plane strains in monolayer $\mathrm{MoS}_{2}[16,46]$.

In addition to orbital composition, it is instructive to investigate the spatial natures of the states. As the second 

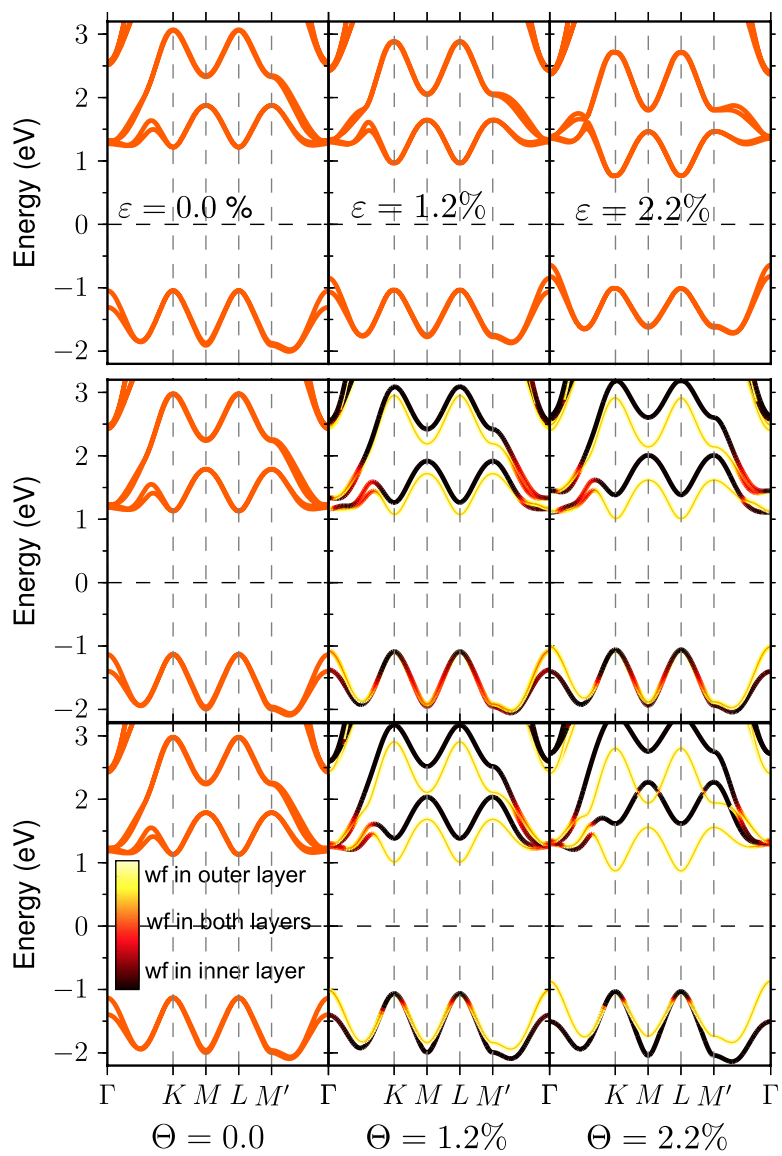

FIG. 2 (color online). Effect of stretching and bending on bilayer $\mathrm{MoS}_{2}$ band structure. Flat bilayer under equibiaxial strains $\varepsilon$ (top row), bilayer under cylindrical bending (middle row), and bilayer under spherical bending (bottom row) at values of $\Theta$ indicated at bottom. Bands are colored by the degree of wave function localization to outer or inner layers, as obtained from Mulliken population analysis.

central result we find that, although initially states are delocalized across both layers, upon bending most of them localize on separate layers (Fig. 2 middle and bottom rows). The localization is particularly pronounced near the $\mathrm{CB}$ edge at $K\left(L^{\prime}\right) / K^{\prime}(L)$ where also the strain-induced energy splittings are significant. Note that localization can still be observed near the VB edge at $K\left(L^{\prime}\right) / K^{\prime}(L)$ regardless of negligible energy splitting. This means that it is now possible to define layer-specific direct band gaps [at $\left.K\left(L^{\prime}\right) / K^{\prime}(L)\right]$ and layer-specific indirect band gaps (between the VB at $\Gamma$ and $\mathrm{CB}$ at $K\left(L^{\prime}\right) / K^{\prime}(L)$ ), both of which systematically decrease and increase with tensile and compressive layer-specific strains. The computed layerspecific direct gaps at $K\left(L^{\prime}\right) / K^{\prime}(L)$ are displayed in Fig. 3; as seen, the gaps in each layer are in close quantitative agreement with the DFTB direct gap at the $K / K^{\prime}$ point of a monolayer under in-plane, equibiaxial strain. Thus, these results reveal that bending not only alters the fundamental band gap of the bilayer but also decouples the layers at the

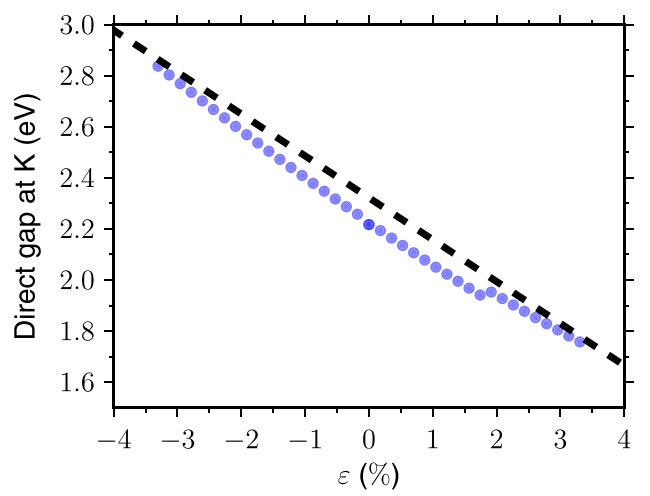

FIG. 3 (color online). Calculated layer-specific direct band gaps (filled symbols) at $K\left(L^{\prime}\right) / K^{\prime}(L)$ as a function of equibiaxial strain within each layer of bilayer $\mathrm{MoS}_{2}$ subjected to spherical bending. The outer and inner layers experience equibiaxial tensile and compressive strains of magnitude $\epsilon= \pm \Theta$, respectively, as noted in the text. The dashed line is the DFTB direct gap at $K / K^{\prime}$ of monolayer $\mathrm{MoS}_{2}$ under equibiaxial strain (no curvature).

$K\left(L^{\prime}\right) / K^{\prime}(L)$ valleys and consequently allows for tuning of the gaps of each layer simultaneously.

Based on the observed robustness of bending-induced decoupling, we now propose a strategy to control valley polarization in bent bilayer $\mathrm{MoS}_{2}$ (Fig. 4). For monolayer $\mathrm{MoS}_{2}$, it is well known that optical transitions at the $K$ and $K^{\prime}$ valleys are governed by the selection rule $\Delta m_{j}= \pm 1$ and, as the rule is valid over a wide region of the surrounding reciprocal space, it also carries over to the band-edge (spin-orbit split) $A$ and $B$ excitons [21,22]. While in a monolayer spin and valley are coupled, in a bilayer they are decoupled due to inversion symmetry, and excitation by circularly polarized light can only control the net spin but not the valley population [21]. This lack of control will persist in bilayers under in-plane equibiaxial strain, where only the overall band gap changes [Figs. 4(a) and 4(b)]; this band-gap change is manifested experimentally as a shift in the absorption and photoluminescence peaks $[13,14]$. However, now that bending breaks inversion symmetry and decouples the layers, could valley-selective physics be realized in these decoupled monolayers? The answer depends on spin-orbit (SO) splitting of the band edges, which is absent in our implementation of DFTB. It is nevertheless possible to advance general arguments to address this issue by using previous works. First, for monolayer $\mathrm{MoS}_{2}$, strain-induced changes in SO splitting of the VB edge at $K / K^{\prime}$ have been shown to be more than an order of magnitude smaller than changes in the band gaps [47]. Our own DFT studies corroborate this finding for both strained monolayers and bilayers (see Supplemental Material [35]). Second, previous DFT calculations by one of us [11] have shown electronic decoupling of layers in bilayer $\mathrm{MoS}_{2}$ under an external electric field, with similar inversion-symmetry breaking. An examination of the SO splitting of the VB edge shows that with decreasing 
(a)

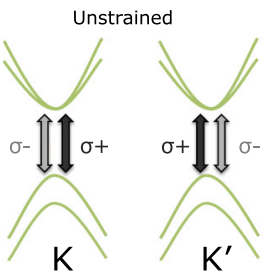

(c)

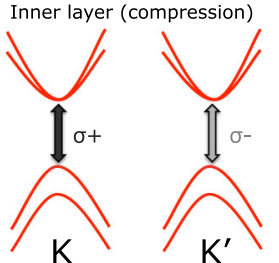

(b)

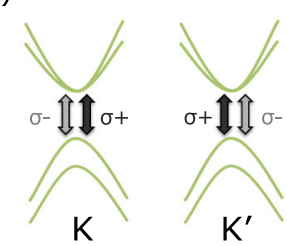

Outer layer (tension)

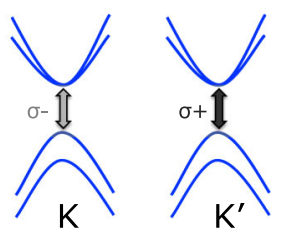

FIG. 4 (color online). Schematic of the proposal to control valley polarization in bilayer $\mathrm{MoS}_{2}$. By optical selection rules, (a) in the absence of strain and (b) in the presence of in-plane, equibiaxial tensile strain, circularly polarized light $(\sigma-$ and $\sigma+)$ populates both $K$ and $K^{\prime}$ valleys. (c) Bending breaks the inversion symmetry and layer degeneracies; hence, time-reversal symmetry again couples spin and valley in each individual layer and circularly polarized light can be used to excite a given valley (by choosing the photon helicity $\sigma$ ) in a given layer (by choosing the photon energy). Spin-orbit splitting of the band edges has been indicated here as a perturbation to the strained band structure.

interlayer coupling (i.e., increasing electric field), the SO splitting of the VB edge for each layer at $K / K^{\prime}$ approaches that of a monolayer (see Supplemental Material [35]). We therefore infer, albeit indirectly, that SO splitting of the VB edge at $K\left(L^{\prime}\right) / K^{\prime}(L)$ will likely persist under the combination of in-plane strains and layer decoupling as induced by bending, leading to the reappearance of valley polarization in each layer. Unlike the case of symmetry breaking by external electric fields where the states from each layer are nearly rigidly translated in opposite directions [11], the band edges of each layer in the bent bilayers move towards or away from each other depending upon the layer-specific strain. Thus, it should further be possible to address a specific layer and a specific valley by selecting both the helicity and the energy of the photon [Fig. 4(c)]. In practice, larger curvatures should induce larger energy differences between layer-specific transitions, thus allowing for the absorption peaks of circularly polarized light to become more pronounced and differentiable. Our proposal is complementary to the approach of Castellanos-Gomez et al. [48] where interlayer coupling in folded $\mathrm{MoS}_{2}$ was reduced by stacking disorder that includes regions of orientational twist between adjacent $\mathrm{MoS}_{2}$ layers. These twisted regions were shown to exhibit enhanced photoluminescence in conjunction with both neutral and charged excitons, characteristic of monolayer $\mathrm{MoS}_{2}$ [49].

In summary, we have shown that bending-induced strain gradients can induce complete decoupling of layers around $K\left(L^{\prime}\right)$ and $K^{\prime}(L)$ valleys in bilayer $\mathrm{MoS}_{2}$, thus rendering

individual sheets as electronically independent monolayers at these valleys with properties governed by layer-specific strains. We propose that this phenomenon could allow for selective pumping of individual $K$ and $K^{\prime}$ valleys of specific layers by selecting the helicity and energy of the exciting laser. Because the electronic structures and electromechanics are similar across the entire family of Group-VI TMDCs, we expect our results and proposals on $\mathrm{MoS}_{2}$ to be broadly applicable and realizable in other Mo and $\mathrm{W}$ dichalcogenides.

P. K. acknowledges the Academy of Finland for funding and the Finnish IT Center for Science (CSC) for computational resources. I. F. and A. R. acknowledge support from the National Science Foundation through the University of Massachusetts, Amherst Materials Research Science and Engineering Center (MRSEC) on Polymers under Award No. DMR-0820506.

*pekka.koskinen@iki.fi †ashwin@engin.umass.edu

[1] K. S. Novoselov, D. Jiang, F. Schedin, T. J. Booth, V. V. Khotkevich, S. V. Morozov, and A. K. Geim, Proc. Natl. Acad. Sci. U.S.A. 102, 10451 (2005).

[2] H. S. S. Ramakrishna Matte, A. Gomathi, A. K. Manna, D. J. Late, R. Datta, S. K. Pati, and C. N. R. Rao, Angew. Chem., Int. Ed. Engl. 49, 4059 (2010).

[3] M. Chhowalla, H. Shin, G. Eda, L. Li, K. Loh, and H. Zhang, Nat. Chem. 5, 263 (2013).

[4] Q. H. Wang, K. Kalantar-Zadeh, A. Kis, J. N. Coleman, and M. S. Strano, Nat. Nanotechnol. 7, 699 (2012).

[5] A. K. Geim and I. V. Grigorieva, Nature (London) 499, 419 (2013).

[6] K. F. Mak, C. Lee, J. Hone, J. Shan, and T. F. Heinz, Phys. Rev. Lett. 105, 136805 (2010).

[7] A. Splendiani, L. Sun, Y. Zhang, T. Li, J. Kim, C. Chim, G. Galli, and F. Wang, Nano Lett. 10, 1271 (2010).

[8] B. Radisavljevic, A. Radenovic, J. Brivio, V. Giacometti, and A. Kis, Nat. Nanotechnol. 6, 147 (2011).

[9] B. Radisavljevic, M. B. Whitwick, and A. Kis, ACS Nano 5, 9934 (2011).

[10] A. Ramasubramaniam, Phys. Rev. B 86, 115409 (2012).

[11] A. Ramasubramaniam, D. Naveh, and E. Towe, Phys. Rev. B 84, 205325 (2011).

[12] L. Kou, T. Frauenheim, and C. Chen, J. Phys. Chem. Lett. 4, 1730 (2013).

[13] K. He, C. Poole, K. Mak, and J. Shan, Nano Lett. 13, 2931 (2013).

[14] H. J. Conley, B. Wang, J. I. Ziegler, R. F. Haglund, Jr., S. T. Pantelides, and K. I. Bolotin, Nano Lett. 13, 3626 (2013).

[15] E. Scalise, M. Houssa, G. Pourtois, V. Afanas'ev, and A. Stesmans, Nano Res. 543 (2012).

[16] P. Johari and V. B. Shenoy, ACS Nano 65449 (2012).

[17] H. Peelaers and C. G. van de Walle, Phys. Rev. B 86, 241401 (2012).

[18] M. Ghorbani-Asl, N. Zibouche, M. Wahiduzzaman, A. F. Oliveira, A. Kuc, and T. Heine, Sci. Rep. 3, 2961 (2013). 
[19] D. Xiao, G.-B. Liu, W. Feng, X. Xu, and W. Yao, Phys. Rev. Lett. 108, 196802 (2012).

[20] H. Zeng, J. Dai, W. Yao, D. Xiao, and X. Cui, Nat. Nanotechnol. 7, 490 (2012).

[21] K.-F. Mak, K. He, J. Shan, and T. F. Heinz, Nat. Nanotechnol. 7, 494 (2012).

[22] T. Cao et al., Nat. Commun. 3, 887 (2012).

[23] A. M. Jones et al., Nat. Nanotechnol. 8, 634 (2013).

[24] S. Wu et al., Nat. Phys. 9, 149 (2013).

[25] Z. Gong, G.-B. Liu, H. Yu, D. Xiao, X. Cui, X. Xu, and W. Yao, Nat. Commun. 4, 1 (2013).

[26] P. San-Jose, E. Prada, E. McCann, and H. Schomerus, Phys. Rev. Lett. 102, 247204 (2009).

[27] H. K. Min, G. Borghi, M. Polini, and A. H. MacDonald, Phys. Rev. B 77, 041407(R) (2008).

[28] L. D. Landau and E. M. Lifshitz, Theory of Elasticity (Oxford, New York, 1986), 3rd ed.

[29] D. Porezag, T. Frauenheim, T. Köhler, G. Seifert, and R. Kaschner, Phys. Rev. B, 51, 12947 (1995).

[30] G. Seifert, H. Terrones, M. Terrones, G. Jungnickel, and T. Frauenheim, Phys. Rev. Lett. 85, 146 (2000).

[31] D. Y. Qiu, F. H. da Jornada, and S. G. Louie, Phys. Rev. Lett. 111, 216805 (2013).

[32] H. Shi, H. Pan, Y.-W. Zhang, and B. I. Yakobson, Phys. Rev. B 87, 155304 (2013).

[33] N. Zibouche, A. Kuc, and T. Heine, Eur. Phys. J. B 85, 49 (2012).

[34] M. Ghorbani-Asl, S. Borini, A. Kuc, and T. Heine, Phys. Rev. B 87, 235434 (2013).

[35] See Supplemental Material at http://link.aps.org/ supplemental/10.1103/PhysRevLett.112.186802, which includes Refs. [50-53].
[36] T. Dumitrică, and R. D. James, J. Mech. Phys. Solids 55, 2206 (2007).

[37] P. Koskinen and O. O. Kit, Phys. Rev. Lett. 105, 106401 (2010).

[38] P. Koskinen and V. Mäkinen, Comput. Mater. Sci. 47, 237 (2009).

[39] I. Milošević, B. Nikolić, E. Dobardžić, M. Damnjanović, I. Popov, and G. Seifert, Phys. Rev. B, 76, 233414 (2007).

[40] O. O. Kit, L. Pastewka, and P. Koskinen, Phys. Rev. B 84, 155431 (2011).

[41] P. Koskinen and O. O. Kit, Phys. Rev. B 82, 235420 (2010).

[42] P. Koskinen, Phys. Rev. B 82, 193409 (2010).

[43] L. Mattheiss, Phys. Rev. B 83719 (1973).

[44] A. Kormányos, V. Zólyomi, N. Drummond, P. Rakyta, G. Burkard, and V. Fal'ko, Phys. Rev. B 88045416 (2013).

[45] T. Li, J. Galli, J. Phys. Chem. C 111, 16192 (2007).

[46] The effect from curvature alone is negligible because even the smallest radii of curvatures are above $\sim 100 \AA$.

[47] T. Cheiwchanchamnangij, W. R. L. Lambrecht, Y. Song, and H. Dery, Phys. Rev. B 88, 155404 (2013).

[48] A. Castellanos-Gomez, H. S. van der Zant, and G. A. Steele, arXiv:1309.4433.

[49] K. F. Mak, K. He, C. Lee, G. H. Lee, J. Hone, T. F. Heinz, and J. Shan, Nat. Mater. 12, 207 (2013).

[50] G. Kresse and J. Furthmüller, J. Comput. Math. Sci. Teach. 6, 15 (1996); Phys. Rev. B 54, 11169 (1996).

[51] J. Klimeš, D. R. Bowler, and A. Michaelides, Phys. Rev. B 83, 195131 (2011).

[52] P. E. Blöchl, Phys. Rev. B 50, 17953 (1994).

[53] G. Kresse and D. Joubert, Phys. Rev. B 59, 1758 (1999). 